a cause of the tumors, but that it likewise is a result of the disturbed ovarian function, as is the marked delay and irregularity in the onset of the menopause, which is often seen in these cases.

Dissection of the Ureter in the Radical Operation for Uterine Carcinoma.-MaYer (Zeitschr. f. Geb. u. Gyn., 1914, lxxv, 399) says that while in many cases the ureter is easily found by the process of separating from above the layers of the broad ligament, in occasional instances this becomes very difficult, especially in the presence of senile or inflamed tissues. Moreover, at times numerous branching veins are present throughout the whole of the broad ligament, and give rise to exceedingly annoying hemorrhage as they are ruptured in separating the layers. In such cases, he has found the following slight modification of the usual technique of great service: The ureter can almost always be distinctly seen shining through its peritoneal covering in the higher portion of the posterior pelvic wall, or at the point where it crosses the great vessels. If the operator, after severing the round ligament and adnexa, draws the uterus forcibly forward and to the opposite side, before any dissection has been done to disturb the natural relations, it is always easy to trace the ureter down by sight to its point of entrance into the parametrium. If a small longitudinal incision be made in the peritoneum at this point, immediately over the ureter, the latter will be exposed without having separated any of the tissues of the broad ligament, and therefore without the production of any hemorrhage. If now the ureter is lifted by means of a long blunt hook, or similar instrument, to one side or the other, the entire posterior layer of the broad ligament can be cut through low down, exposing the uterine artery and ureter in their entire course through the ligament, without the necessity for any blunt dissection of tissue. By this means, it is possible to remove a larger amount of paremetrium than by the usual methods, and the uterine artery is easily exposed for ligation, without any danger to the ureter.

Action of Ovarian Lipoids upon the Genital System.-An interesting report has recently been made by Iscovesco (Rev. de gyn. et de chir., abdom., 1914, xxii, 161) upon some experimental and clinical studies of certain lipoids obtained from human ovaries. As Iscovesco points out, every gland of internal secretion throws into the circulation a number of different lipoidal substances, one of which appears to have the specific action of stimulating the secretory activity of the gland itself. Fresh human ovary was found to contain about 3 per cent. by weight of total lipoids; extracting these by means of ether, acetone, chloroform, alcohol, etc., Iscovesco was able to distinguish five main groups, of which the fifth, or alcohol-soluble group is much the most important. By further solution and precipitation, this group can be subdivided into several different constituents, one of which, called by Iscovesco VDc, appears to exert a marked stimulant acting upon the ovary and whole genital system. It is a yellowish, wax-like substance; which for experimental and clinical use was made up in a 2 per cent. solution in oil. This solution may be injected hypodermically or intraperitoneally into laboratory animals, without any signs of irritation or infection. A series of young rabbits injected daily, or at inter- 
vals of a few days, for a considerable period of time, all showed when killed and autopsied an enlargement of the ovaries and uterus to two or three times the size of these organs in controls; very young animals, in whom the injections were started at the sixth week and continued until the fifteenth week, showed by that time genital organs exceeding in size and weight those of adult animals. Practically no effect was produced in any of the experiments on any of the other organs, except the thyroid, which showed in the injected animals a slight increase in size; the effect here was much less, however, than in the genital tract. Iscovesco thinks the substance acts by direct stimulation of the genital centres in the central nervous system. For therapeutic purposes the same oily solution was given in 1 c.c. doses by deep injection into the gluteal muscles once daily, or four to six pills, each containing $2 \mathrm{cgm}$. of the lipoid, were given by mouth. The author reports astonishingly good results in the treatment of a number of cases of dysmenorrhea, amenorrhea due to ovarian hypofunction, and various nervous conditions in young girls, associated with irregular or painful menstruation, and probably having a basis in disturbed ovarian activity. Equally good results were obtained from the treatment of a number of patients suffering from the results of a natural or artificial menopause. Iscovesco does not think that the corpus luteum is responsible for the secretion of any substance essentially different from that elaborated by the entire ovary; if there is any difference, it is merely that the active substanccs are present in the corpus luteum in greater concentration. He has isolated from the corpus luteum a lipoid possessing identical chemical and physical characteristics with the one obtained from the ovary, and in a small number of animal experiments and therapeutic tests the results have been entirely similar.

\section{DER MATOLOGY}

UNDER THE CHARGE OF

MILTON B. HARTZELL, M.D.,

PROFESSOR OF DERMATOLOGY IN THE UNIVERSITY OF PENNSYLVANIA.

\section{Disseminated Hematogenous Tuberculosis Verrucosa Cutis.-} Bourgeols (Dermatol. Zeitsch., Band xxi, Heft 1), who has made a most careful study of two cases of verrucose tuberculosis of the skin with multiple disseminated lesions, following, in the one instance measles, in the other scarlet fever, concludes that, besides the ordinary form which arises from local infection, there is a second; hematogenous in origin. The hematogenous form occurs chiefly in children with or without previous tuberculous symptoms, almost always after some one of the exanthemas, usually measles, much less frequently scarlet fever, and is not infrequently associated with other forms of cutaneous tuberculosis, such as the papulonecrotic tuberculide and lichen scrofulosorum. Clinically and histologically the hematogenous variety 